\title{
Experiencias adaptativas de mujeres mastectomizadas: una mirada desde el Modelo de Callista Roy
}

Adaptive experiences of women with mastectomies:

A view from the Model Callista Roy

Experiências adaptativas de mulheres mastectomizadas: Uma olhada desde o Modelo de Callista Roy

Ian Coahtepetzin Zavala-Pérez ${ }^{1}$; Cecilia Palacios-Fonseca²; Cinthia Viridiana Olea-Gutiérrez ${ }^{3}$; Daniela Lizbeth Salas-Medina ${ }^{4}$; Martha Xitlali Mercado-Rivas ${ }^{5}$

${ }^{1}$ Maestro en Enfermería. Asesor(a) en tanatología y estudiante de psicología. Correo electrónico: ic_jm82@hotmail.com

${ }^{2}$ Maestra en Enfermería. Asesora en tanatología y estudiante de psicología. Correo electrónico: cepalfo_05@hotmail.com

${ }^{3}$ Maestra en Enfermería y estudiante del Doctorado en Ciencias de Salud Pública de la Universidad de Guadalajara. Correo electrónico: Viri_olea82@hotmail.com

${ }^{4}$ Daniela Lizbeth Salas-Medina. Maestra en Administración y Doctora en Psicología. Correo electrónico: deptopsicoenfria@gmail.com

${ }^{5}$ Martha Xitlali Mercado-Rivas. Maestra en Terapia Familiar Sistémica y Doctora en Psicología. Universidad Autónoma de Nayarit, Área de Ciencias de la Salud. Nayarit, México. Cuerpo Académico de Psicología de la Salud en la línea de Psicología, Salud y Enfermedad. Correo electrónico:

dpp.uam0@gmail.com

Cómo citar este artículo en edición digital: Zavala-Pérez, I.C., Palacios-Fonseca, C., Olea-Gutiérrez,

C.V., Salas-Medina, D.L. E Mercado-Rivas, M.X. (2019). Experiencias adaptativas de mujeres mastectomizadas: Una mirada desde el Modelo de Callista Roy. Cultura de los Cuidados (Edición digital), 23(53). Recuperado de http://dx.doi.org/10.14198/cuid.2019.53.05

Correspondencia: Mtro. Ian Coahtepetzin Zavala Pérez. C.P. 63000; tel. 311123 2008; calle Oaxaca \# 77

Colonia Centro. (México)

Correo electrónico de contacto: ic_jm82@hotmail.com

Recibido: 10/11/2018; Aceptado: 08/02/2019

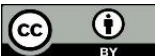

\section{ABSTRACT}

Objective: To understand the basis of the Callista Roy Adaptation Model, adaptive experiences of women who have undergone a mastectomy.

Methods: Phenomenological methodology was the selected path. Data were collected through a depth interview and presented in the form of a preliminary script of open questions grounded in the Roy Adaptation Model. For the organization and management of data was used the Colaizzi method. The study was developed in the State Cancer Center of Nayarit. 
Results: Alterations suffered by women with mastectomies, include those concerning the adaptation modes defined by Roy Theory, which are physiological, selfconcept, role function and interdependence.

Conclusions: The loss of one or both breasts for women represents a major change in their lives, which may affect, the way she perceives herself, her system of beliefs and values. Adaptation experiences identified through the responses of the participants, most of them attributed to religion, as an important motivation to continue their lives.

Keywords: Women with mastectomies, cancer, adaptation, Roy.

\section{RESUMO}

Objetivo: Compreender com base no Modelo de Adaptação de Callista Roy, as experiências de adaptação de mulheres submetidas à mastectomia.

\section{Metodologia: Seleccionou-se a} metodologia de trajetória fenomenológica. Os dados foram coletados por meio de uma entrevista à profundidade e apresentou-se na forma de questões abertas com aterradas no Modelo de Adaptação de Callista Roy. No estudo foi utilizado o metodo de Colaizzi para a organização e gestão dos dados. $\mathrm{O}$ espaço em que foi desenvolvido o estudo, foi o Centro Estatal de Cancerologia de Nayarit.

Resultados: Foram detectados alterações nos modos de adaptação definidos pela teoria de Roy: fisiológico, auto-conceito, função do rol e interdependência.

Conclusões: A perda de um ou ambos seios, representa uma grande mudança nas suas vidas, na cual podem ser afetadas as maneiras de autopercepção, sistema de crença e valores. As experiências de adaptação identificadas através das respostas das participantes na maioria delas são atribuídas à religião como um fator motivador para continuar com as suas vidas.

Palavras chave: Mulheres mastectomizadas, câncer, adaptação, Roy.

\section{RESUMEN}

Objetivo: Comprender con base en el Modelo de Adaptación de Callista Roy, las experiencias adaptativas de las mujeres sometidas a mastectomía.

Métodos: Se seleccionó la metodología de trayectoria fenomenológica. Los datos fueron recogidos a través de una entrevista a profundidad y se presentó bajo la forma de un guion preliminar de preguntas abiertas fundamentadas en el Modelo de Adaptación de Roy. En el estudio se empleó el método de Colaizzi para la organización y manejo de los datos. El espacio en el que se desarrolló el estudio fue en el Centro Estatal de Cancerología de Nayarit.

Resultados: Se detectaron alteraciones en los modos de adaptación definidos por la teoría de Roy: fisiológico, auto-concepto, función del rol e interdependencia.

Conclusiones: La pérdida de una o ambas mamas para la mujeres representa un gran cambio en sus vidas, en el cual pueden verse afectadas las maneras de autopercepción, sistema de creencias y valores. Las experiencias de adaptación identificadas, a través de las respuestas de las participantes en su mayoría se atribuyen a la religión como un factor de motivación para continuar con sus vidas.

Palabras clave: Mujeres mastectomizadas, cáncer, adaptación, Roy.

\section{INTRODUCCIÓN}

Según la Organización Mundial de la Salud, define al cáncer como un proceso de crecimiento y diseminación incontrolados de células; el tumor suele invadir el tejido 
circundante y puede provocar metástasis en puntos distantes del organismo (Organización Mundial de la Salud [OMS], 2016). El cáncer de mama es una enfermedad multifactorial en la que los factores genéticos y ambientales contribuyen a su aparición (Dornelles Prolla, Santos da Silva, Oliveira Neto, Goldim \& Asthon-Prolla, 2015).

En el ámbito mundial, el cáncer de mama es la segunda neoplasia más frecuente en la población y la más frecuente entre las mujeres con un estimado de 1.67 millones de nuevos casos diagnosticados anualmente, representando el 25\% de los casos de cáncer en mujeres. La incidencia es más alta en países desarrollados (excepto Japón); varía desde 27 por 100,000 mujeres en África Central, hasta 96 en Europa occidental (Secretaria de Salud [SSA], 2015).

Es también la principal causa de muerte por un tumor maligno en la mujer en países en vías de desarrollo y la segunda en países desarrollados (después del cáncer de pulmón) con una defunción cada minuto por esta causa en alguna parte del mundo $(522,000)$ y tasas de mortalidad que van de 6 en Asia oriental a 20 defunciones por 100,000 mujeres en África Occidental (SSA, 2015). La tendencia de la mortalidad es ascendente debido a una mayor incidencia de la enfermedad, el envejecimiento poblacional y la poca capacidad de respuesta de los sistemas de salud en países subdesarrollados (SSA, 2015). En América Latina, el cáncer de mama es el cáncer más frecuente con 152,059 casos diagnosticados anualmente, una cuarta parte $(24.9 \%)$ de los casos de cáncer en mujeres. La incidencia regional es 47.2, la cual es más alta en países del cono sur, principalmente Argentina y Uruguay, donde las tasas son semejantes a la de países desarrollados (71.2 y 69.7 defunciones por 100,000 mujeres, respectivamente) (SSA, 2015).

En México, a partir del año 2006, el cáncer de mama ocupa el primer lugar como causa de muerte por neoplasia maligna en mujeres de 25 años en adelante y ha desplazado de esta posición al cáncer cervicouterino. En el año 2010 se registraron 5,113 defunciones, con una tasa de mortalidad de 10.1 fallecimientos por 100 mil mujeres, lo que hace un total de 13,648 nuevos casos (Robles Castillo, Ruvalcaba Limón, Maffuz \& Rodríguez Cuevas, 2011).

En el año 2013, se registraron 5,405 defunciones en mujeres con una tasa de 16.3 defunciones por 100,000 mujeres. Las entidades con mayor mortalidad por cáncer de mama son Coahuila (24.2), Sonora (22.6) y Nuevo León (22.4).

En Nayarit, región donde se desarrolla esta investigación, en el año 2010 según datos del Instituto Nacional de Estadística, Geografía e Informática, la mortalidad por cáncer de mama ya representaba un $11.5 \%$ de todas las muertes por cáncer (Instituto Nacional de Estadística, Geografía e Informática [INEGI], 2012).

El cáncer de mama es sin duda una enfermedad relevante para la salud pública por afectar profundamente el bienestar físico, mental y social de la mujer. Además de traer repercusiones negativas como depresión, angustia, miedo de morir, inseguridad, perjudicando la auto-imagen y auto-estima de las mujeres tratadas (García Sánchez, Insuasty Enríquez \& Parra Gómez, 2011).

\section{Un modelo explicativo}

La necesidad de entender el fenómeno que las mujeres viven tras ser mastectomizadas, ha motivado a utilizar un modelo teórico que permita valorar a la persona como un todo, así mismo permita conocer sus experiencias surgidas durante su proceso de 
adaptación. Por tal motivo debido a los supuestos filosóficos que se plantean en el Modelo de Adaptación de Callista Roy, permiten su utilización como base científica que guie el proceso de comprensión de las experiencias adaptativas de las mujeres mastectomizadas.

"Roy describe a las personas como seres holísticos, con partes que funcionan como unidad con algún propósito, no en una relación causa-efecto. Los sistemas humanos incluyen a las personas como individuos, grupos, familias, comunidades, organizaciones, y a la sociedad como un todo" (Díaz de Flores et al., 2009).

Roy plantea que las personas, vistas como sistemas adaptativos holísticos, se encuentran en continua interacción con un ambiente cambiante. Los estímulos ambientales, al entrar en contacto con el sistema, activan los subsistemas de afrontamiento regulador y cognitivo $\mathrm{y}$ desencadenan una serie de respuestas observables a través de los cuatro modos de adaptación (fisiológico, auto concepto, función del rol e interdependencia); estas respuestas buscan afrontar la situación y, de esta manera, promover la adaptación (Gutiérrez, 2007).

El ambiente es todo aquello que rodea a la persona y lo que está dentro de ella. El ambiente no se limita al entorno, está constituido además por sus experiencias y los aspectos que constituyen su ambiente interno (Díaz de Flores et al., 2002).

Roy señala que en el ambiente se encuentran los estímulos, los cuales clasifica en focales, contextuales y residuales:

- Los estímulos focales son aquellas situaciones o circunstancias que la persona debe confrontar en forma inmediata, tales como una enfermedad o un evento externo, y para lo cual requiere consumo de energía.

- Los contextuales son otros estímulos que están presentes en la situación, y aunque no son el centro de atención o de consumo de energía, influyen en la forma como la persona puede afrontar el estímulo focal.

- Los estímulos residuales son factores desconocidos que se encuentran en el ambiente, cuyos efectos no han sido confirmados. Estos pueden ser una expectativa, un valor, una actitud o una creencia, producto de experiencias pasadas. En el momento en que se conoce como están actuando en la situación, se vuelven contextuales (Gutiérrez, 2007; Melo, da Silva, Mota, Mamede, Lindard \& Fernández, 2011).

Estos estímulos activan los subsistemas de afrontamiento regulador y cognitivo con el fin de generar en el individuo una respuesta que permita controlar la situación. Por su parte el subsistema de afrontamiento regulador, está dirigido por los sistemas nerviosos autónomos y endocrinos, y responde de manera automática a los estímulos del ambiente interno y externo. Así mismo el subsistema de afrontamiento cognitivo, se rige por la conciencia y tiene relación con los procesos cognoscitivos y emocionales que le permiten a la persona interpretar la situación y afrontar los estímulos ambientales con base en su análisis de la situación, fundamentada en su experiencia y educación (Gutiérrez, 2007; Melo et al., 2011).

Las respuestas de los individuos se evidencian en forma interrelacionada en cuatro modos de adaptación (Gutiérrez, 2007; Melo et al., 2011):

- El fisiológico se relaciona con las respuestas fisiológicas a los estresores o 
estímulos externos.

- El auto concepto representa la salud emocional, se enfoca en las dimensiones espirituales y psicológicas de la persona.

- La función del rol se relaciona con el papel que desempeña una persona en la estructura social.

- La interdependencia tiene que ver con las relaciones afectivas y sociales que se establecen como personas significativas y sistemas de apoyo.

\section{La comprensión de las experiencias vividas}

La enfermería necesita identificarse con una filosofía que le permita otorgar un sentido interpretativo a los fenómenos individuales con el fin de determinar la importancia de los cuidados en las experiencias de salud o enfermedad (Contreras \& Castillo, 2016).

La investigación cualitativa es una perspectiva que ofrece los materiales y métodos necesarios para que el profesional de enfermería lleve a cabo la interpretación de los significados bajo un análisis crítico y reflexivo (Bueno, 2011).

Así mismo, la investigación cualitativa ofrece una metodología de investigación que permite generar conocimiento como una creación compartida entre el investigador y las mujeres investigadas. Cabe mencionar, que no se pretende medir la realidad sino adentrarse en ella para comprenderla (Contreras \& Castillo, 2016).

Habría que decir también, que el investigador dentro de la perspectiva cualitativa se caracteriza porque es considerado como instrumento para la colecta de datos y participe de la vida de otros, se enfoca en el estudio de los procesos y de los significados; así mismo, se interesa por las experiencias humanas y considera la subjetividad e intersubjetividad elementos esenciales para desarrollar conocimiento (Baptista, Merighi \& Freitas, 2012).

Considerando lo anterior, el presente estudio tiene como objetivo describir con base en el Modelo de Adaptación de Callista Roy, las experiencias adaptativas de las mujeres sometidas a mastectomía en los aspectos de autopercepción, roles y afectos en contexto socio-familiar.

\section{METODOLOGÍA}

Planteamos una perspectiva metodológica cualitativa. El propósito de la investigación fenomenológica es describir las experiencias tal como son vividas por los individuos mujeres mastectomizadas-. Además, se hace énfasis en la comprensión de lo que una experiencia puede representar dentro del contexto de vida de las personas, lo que es denominado como capturando la experiencia vivida (Contreras \& Castillo, 2016).

De igual modo, la fenomenología descriptiva, se interesa por la forma en cómo se adquiere el conocimiento, pues hace énfasis en describir la experiencia vivida a través de la corporalidad tal y como se introyecta en la conciencia; se propone la intuición como rasgo humano para develar los significados a través de la reducción fenomenológica, porque afirma que el conocimiento yace en la conciencia del ser (Gill, 2014).

\section{Material y métodos}

Se abordaron seis mujeres mastectomizadas de la ciudad de Tepic Nayarit, que recibían quimioterapia en el Centro Estatal de Cancerología. La estrategia de muestreo para la selección de las participantes fue propositivo (Teddlie y $\mathrm{Yu}$, 2007). Para la muestra se consideró a 
mujeres con un rango de edad de 45 a 63 años, no se consideró la ocupación; sin embargo, todas las participantes fueron amas de casa.

A las participantes se les explicó el objetivo del estudio y enfatizó que las entrevistas serían grabadas; así mismo, que podría ser suspendida en cualquier momento que lo pidieran. En la misma tónica, se les informó que los datos obtenidos serían de carácter confidencial y con fines académicos, resguardando sus identidades. Todas las entrevistas fueron transcritas en los días siguientes (10 días consecutivos) a su grabación. Para identificar las entrevistas, se les asigno un número a manera de código ejemplo: $\mathrm{m} 01, \mathrm{~m} 02, \mathrm{~m} 03$, entre otros.

Los datos fueron recogidos a través de una entrevista a profundidad, cuyo objetivo fue ampliar al máximo las posibilidades de captación del proceso, y se presentó bajo la forma de un guion preliminar de preguntas abiertas fundamentadas en el Modelo de Adaptación de Roy con la pregunta inicial ¿Cómo ha sido su vida desde la mastectomía? En el estudio se empleó el método de Colaizzi para la organización y manejo de los datos, dicho método consta de pasos iniciando con la definición del fenómeno de interés, recolección de las descripciones de las participantes sobre el fenómeno, lectura de dichas descripciones y extracción y escritura del significado de cada enunciado significativo, organización de los significados formalizados agregados en grupos de temas y por último, desarrollar una descripción exhaustiva (Sánchez, 2006; Lewis, 2014).

Una vez transcritas las entrevistas, se procedió a realizar lectura y re-lectura de todas las descripciones hechas por las participantes acerca de sus experiencias respecto al procedimiento quirúrgico denominado mastectomía haciendo énfasis en las rutinas y estrategias adaptativas ante dicha situación.

\section{RESULTADOS}

Se extractaron todos los enunciados significativos, frases y planteamientos de cada descripción relacionados directamente con las experiencias de estar y ser mujeres mastectomizadas. Posteriormente, se determinaron los significados y se agruparon en conjunto de significados formulados hasta constituir categorías desde los modos adaptativos: Fisiológico, autoconcepto, función del rol e interdependencia.

Otro rasgo que es importante destacar sobre el método Colaizzi es que después de transcribir línea por línea y extraer enunciados significativos sobre el fenómeno; así como elaborar un significado formulado basado en dichos enunciados; para posteriormente, hacer la descripción exhaustiva; es que ésta descripción se presenta de regreso a las participantes que son consideradas co-investigadores de la validación (ver tabla 1). Por consiguiente, el resultado de la investigación es la descripción del fenómeno a través de estos temas que capturan la esencia de la experiencia. La descripción de estos temas fueron reportados de regreso a tres participantes para validación. De acuerdo a la retroalimentación, no fue necesario hacer modificación al análisis, el cual revela cuatro temas (ver tabla 2).

Entre las alteraciones sufridas por las mujeres mastectomizadas, se detectaron aquellas referentes a los modos de adaptación definidos por la teoría de Roy: fisiológico, auto-concepto, función del rol e interdependencia (ver tabla 3). 


\section{TABLA 1: Extracción de las declaraciones significantes y significados formulados. Ejemplos de enunciados}

\section{DECLARACIONES SIGNIFICANTES}

"Solo al principio de que me hicieron la operación sentía mucho dolor"

"Al mover mi brazo derecho un tiempo sentí un pequeño dolor"

"Cuando de recién de la cirugía si sentía que me iba de lado al caminar"

\section{SIGNIFICADOS FORMULADOS}

La paciente refiere dolor, el cual como en toda cirugía se presenta.

El dolor se presenta como consecuencia de las actividades realizadas por la paciente.

La pérdida de equilibrio es una de las consecuencias más comunes después de la realización de una mastectomía.

La pérdida de una parte del cuerpo implica un duelo como cualquier otra perdida, y la paciente sentía tristeza por verse sin esa parte de su cuerpo. tristeza y me ponía a llorar'

"Hay momentos que recuerdo o que veo alguna fotografía de cómo me veía anteriormente y todo lo que he pasado y a veces me siento triste"

"Eso te afecta en todo y lo más evidente es lo físico porque te das cuenta que ya no te ves igual, pero el cambio más importante viene siendo en ti misma, en lo que piensas de ti"

"Hay momentos en los que si me afecta pero imagino que es normal, sé que es una parte de mi cuerpo y así perdiera mi mano o una pierna tendría el mismo sentimiento"

"Los primeros meses si cambió mi papel, debido a que me ayudaban a cuidar a mis hijos y también yo recibía cuidados"

"A veces siento que se preocupan demasiado y hacen cosas por mi cosas que antes no hacian"

"Pues si, después de la quimio, me siento débil, así que ahí es cuando me acuesto casi todo el día, porque me siento mareada, no puedo caminar muy bien y a veces me duele la cabeza, esas quimios me ganan, pero luego yo les gano a ellas"

"Soy una persona que lucha por lo que quiere y saca adelante a su familia, me gusta ayudar, me considero amigable pero sobre todo me considero una persona fuerte"
Los recuerdos del aspecto físico anterior a la cirugía causan nostalgia y un sentimiento de tristeza.

El aspecto físico es casi lo más importante para una mujer que ha sido mastectomizada, ya que ellas sienten que la gente ya no las va a ver igual y que las van a discriminar.

Para algunas personas cualquier pérdida es igual, no hay una que afecte más que otra, en este caso la paciente refiere que para ella sería el mismo sentimiento el perder cualquier otra parte de su cuerpo.

Se presentan cambios en los roles debido a la incapacidad que se presenta con la cirugía

Cuando a alguien presenta alguna enfermedad, las personas que la rodean se acercan más a ella y tratan de hacerla sentir mejor.

Dificultades fisiológicas presentadas por la paciente posterior a la mastectomía afectan el campo de energía. 
TABLA 2: Nodos de Sentido desde los modos de adaptación

\begin{tabular}{|c|c|c|}
\hline SIGNIFICADOS FORMULADOS & GRUPO DE TEMAS & TEMA \\
\hline $\begin{array}{l}\text { El dolor se presenta como consecuencia } \\
\text { de las actividades realizadas por la } \\
\text { paciente. } \\
\text { La pérdida de equilibrio es una de las } \\
\text { consecuencias más comunes después de la } \\
\text { realización de una mastectomía. } \\
\text { Dificultades presentadas por la paciente } \\
\text { fisiológicamente después de la } \\
\text { mastectomía afectan el campo de energía. }\end{array}$ & $\begin{array}{l}\text { Identificación } \\
\text { consecuencias de }\end{array}$ & Fisiológico \\
\hline $\begin{array}{l}\text { La pérdida de una parte del cuerpo } \\
\text { implica un duelo y la paciente sentía } \\
\text { tristeza por verse sin esa parte de su } \\
\text { cuerpo. } \\
\text { Los recuerdos del aspecto físico } \\
\text { anterior a la cirugía causan nostalgia y un } \\
\text { sentimiento de tristeza. } \\
\text { El aspecto físico es importante para una } \\
\text { mujer que ha sido mastectomizada, ellas } \\
\text { sienten que la gente ya no las va a ver igual } \\
\text { y que las van a discriminar. } \\
\text { Para algunas personas cualquier } \\
\text { pérdida es igual, no hay una que afecte más } \\
\text { que otra. } \\
\text { Aceptación del cambio de vida y ser } \\
\text { mujer no se define con tener un cuerpo } \\
\text { perfecto o siquiera completo. } \\
\text { A pesar de que una mujer presenta una } \\
\text { mastectomía no la hace sentirse menos. }\end{array}$ & $\begin{array}{l}\text { Sentimientos y } \\
\text { sensaciones que vienen } \\
\text { después de la pérdida de } \\
\text { una parte del cuerpo. } \\
\text { Percepción que tienen } \\
\text { de sí mismas las mujeres } \\
\text { mastectomizadas. } \\
\text { Afrontamiento de la } \\
\text { mujer en relación a la } \\
\text { pérdida de una mama. }\end{array}$ & Autoconcepto \\
\hline $\begin{array}{l}\text { Se presentan cambios en los roles } \\
\text { debido a la incapacidad que se presenta } \\
\text { con la cirugía }\end{array}$ & $\begin{array}{l}\text { Cambios de actividades } \\
\text { presentes en la vida } \\
\text { cotidiana. }\end{array}$ & Función del rol \\
\hline $\begin{array}{l}\text { Después de la cirugía la paciente } \\
\text { muestra autosuficiencia/dependencia pues } \\
\text { dice ella hacer todo lo que está dentro de } \\
\text { sus capacidades. Aun así, siente no poder } \\
\text { hacer todo como antes lo hacía. } \\
\text { Cuando a alguien se le realiza una } \\
\text { cirugía o que se le presenta alguna } \\
\text { enfermedad, las personas que la rodean se } \\
\text { acercan más a ella y tratan de hacerla sentir } \\
\text { mejor, pero algunas veces esto hace que la } \\
\text { persona enferma sienta que es inútil o que } \\
\text { ya no puede hacer nada. }\end{array}$ & $\begin{array}{lll}\text { Generación } & & \text { de } \\
\text { sentimientos } & & \text { de } \\
\text { dependencia } & \text { y } & \text { de } \\
\text { invalidez } & & \end{array}$ & Interdependencia \\
\hline
\end{tabular}


TABLA 3: Alteraciones adaptativas

\begin{tabular}{|c|c|}
\hline Primer tema: FISIOLÓGICO & Segundo tema: AUTOCONCEPTO \\
\hline Identificación de consecuencias de la cirugía & $\begin{array}{l}\text { Sentimientos y sensaciones que vienen } \\
\text { después de la perdida de una parte del cuerpo }\end{array}$ \\
\hline Dolor & Tristeza \\
\hline Pérdida de equilibrio & Nostalgia \\
\hline Limitación física & Sentimiento de pérdida \\
\hline Cefalea & $\begin{array}{l}\text { Percepción que tienen de sí mismas las } \\
\text { mujeres mastectomizadas }\end{array}$ \\
\hline Mareo & Discriminación \\
\hline Debilidad & Cambio de la autopercepción \\
\hline \multirow[t]{2}{*}{ Cansancio } & $\begin{array}{l}\text { Afrontamiento de la mujer en relación a la } \\
\text { perdida de una mama }\end{array}$ \\
\hline & Sentimiento de pérdida \\
\hline Tercer tema: FUNCIÓN DEL ROL & Cuarto tema: INTERDEPENDENCIA \\
\hline Cambio en el desempeño de la vida cotidiana & $\begin{array}{l}\text { Generación de sentimientos de dependencia y } \\
\text { de invalidez }\end{array}$ \\
\hline Cambios en el rol & Sentimiento de dependencia \\
\hline Cambios en el desempeño & Sentimiento de invalidez \\
\hline Cambios en la vida cotidiana & \\
\hline
\end{tabular}

\section{DISCUSIÓN Y CONCLUSIONES}

La experiencia de pérdida de una o ambas mamas para las mujeres significa un gran cambio en sus vidas, el análisis de dichas experiencias arrojan aspectos como:

- Modos en que ellas se perciben a sí mismas relacionado con el autoconcepto y la autoimagen.

- Estrategias de afrontamiento-adaptación que emergen de las redes de apoyo familiar; así mismas, las prácticas religiosas-espirituales relacionadas con sus creencias y valores.

- Se identifica a la religión como un factor motivador importante para continuar con sus vidas.

- La alteración sufrida por el proceso de mastectomización y sus tratamientos consecuentes se vieron reflejados en los modos de adaptación que define la teoría de Roy como: fisiológico, auto-concepto, función del rol e interdependencia (Melo, Lopes, Araujo \& Gimenez Galvão, 2011). 


\section{Modo Fisiológico}

"Este modo se relaciona con las respuestas fisiológicas a los estresores o estímulos externos" (Rodríguez, Viana \& Andrade, 2015).

En este modo las mujeres mastectomizadas expresaron tener dolor debido a la intervención quirúrgica $\mathrm{y}$ dificultad del movimiento, así como limitación física para realizar actividades cotidianas como el vestir, el baño e higiene personal. También refirieron sentir pérdida del equilibrio.

\section{Modo auto concepto}

"El auto concepto implica una autoevaluación de todo lo que, según el propio individuo, ha sido, es y aspira ser. Incluyen medidas físicas y descripciones del tamaño y forma del cuerpo, así como también los juicios sobre la conveniencia de los propios valores o deseos. Entonces auto concepto es la suma total de las valoraciones y evaluaciones del yo, que representan la propia individualidad. El auto concepto está determinado por las interacciones que mantiene la persona con los demás y las reacciones que presentan los demás frente a éste" (Gutiérrez, 2007).

En sus sistemas de creencias y cómo estas mujeres se ven delante de los otros, atribuyen la motivación para continuar su vida a una fuerza superior. Involucraron a Dios como la fuerza para enfrentar la situación. Se encontró en la religión un apoyo para afrontar el desequilibrio y aumentar la esperanza.

En lo que se refiere a la capacidad de adaptación del individuo a la situación, las mujeres mastectomizadas fueron cuestionadas sobre la adaptación a su problema y a las alteraciones en sus rutinas. Expresan que, a pesar de que sufren por la mutilación de alguna de las mamas, consiguen superar y reconocer que necesitan seguir viviendo una vida "normal".

\section{Modo función de rol}

"El modo de la función de rol se centra en el papel de la persona en la sociedad y los roles dentro de un grupo. La necesidad básica que subyace en el modo de función es la integridad social, es decir, la necesidad de saber quién es uno en relación a los demás por lo que uno va a saber para actuar" (Cutcliffe, McKenna \& Hyrkäs, 2011; Raile Alligood \& Marriner Tomey 2011).

En relación a este modo, las mujeres investigadas refieren un profundo sentido de responsabilidad maternal hacia sus hijos, y en general hacia la familia, reconociendo la importancia de seguir siendo el vínculo que une a la familia.

También mencionan que al asignar prioridades en su vida, pueden dejar en segundo lugar satisfacer sus propias necesidades, en este caso la posibilidad de poder someterse a una reconstrucción mamaria o implantes mamarios, con el fin de restaurar su imagen corporal.

En el ámbito social, la mayoría de las mujeres entrevistadas señalan que no hay cambios significativos en sus vidas pese que fueron mastectomizadas.

\section{Modo de interdependencia}

"La autoimagen y el dominio del papel social de cada individuo interacciona con las personas de su entorno, ejerciendo $\mathrm{y}$ recibiendo influencias. Esto crea relaciones de interdependencia, que pueden ser modificadas por los cambios del entorno" (Cutcliffe, McKenna \& Hyrkäs, 2011; Raile Alligood \& Marriner Tomey 2011).

Para las mujeres entrevistadas con mastectomía, el apoyo de sus amistades y, 
sobre todo, de sus familiares es altamente valorado; es decir, el contar con una red de apoyo en el transcurso del proceso es fundamental ya que contribuye a la satisfacción de las necesidades humanas, sobre todo en procesos de enfermedad como lo es el cáncer de mama (Vivar, 2012).

\section{Consideraciones para el profesional de enfermería}

Hacemos referencia a Campos et al. (2011), citado por Contreras \& Castillo-Arcos (2016) cuando explican que es indispensable que el profesional de enfermería posea el conocimiento para tomar decisiones fundamentadas, en busca de una atención integral e individualizada que valore los significados más íntimos de las personas acerca de la enfermedad, los tratamientos o el contexto en el cual se desarrolla el fenómeno; es decir, "la comprensión de las estructuras del significado de las experiencias vividas".

Acorde con lo anterior, comprender las experiencias de estas mujeres permitiría que el profesional de enfermería llevará a cabo intervenciones focalizadas para dar respuestas a las dimensiones afectadas, a través del diseño de planes de cuidados con enfoques multidisciplinarios que incluyan la valoración del impacto psicosocial de la enfermedad, el seguimiento y control de las secuelas físicas y emocionales, así como de los efectos tardíos potenciales de los tratamientos (Melo et al., 2011 y Vivar, 2012).

Respecto al Estado, éste debería tener una mayor participación en conjunto con la población y grupos de interés a través de articular la participación de las diversas instituciones para lograr acciones coordinadas entre los sectores gubernamentales y no gubernamentales, una adecuada financiación e implementar sistemas de información que permitan tomar decisiones basadas en pruebas científicas.

\section{BIBLIOGRAFÍA}

- Baptista, P., Merighi, M. \& Freitas, G.F. (2012). El estudio de la fenomenología como una vía de acceso a la mejora de los cuidados de enfermería. Cultura de los cuidados, 10(29), 9-15. Recuperado de https://doi.org/10.7184/cuid.2011.29.02.

- Bueno Robles, L.S. (2011). Aspectos ontológicos y epistemológicos de las visiones de enfermería inmersas en el quehacer profesional. Ciencia $y$ enfermería, 17(1), 37-43. Disponible en https://dx.doi.org/10.4067/S0717-95532011000100005.

- Contreras, H.S. \& Castillo, L.C. (2016). Fenomenología: una visión investigativa para enfermería. Cultura de los cuidados, 20(44), 15-24. DOI: https://doi.org/10.14198/cuid.2016.44.02.

- Cutcliffe, J., McKenna, H. \& Hyräs, K. (2011). Modelos de Enfermería, aplicación a la práctica. México: Manual Moderno.

- Díaz de Flores, L., Durán de Villalobos, M.M., Gallego de Pardo, P., Gómez Daza, B., Gómez de Obando, E., González de Acuña \& Venegas Bustos, B.C. (2002). Análisis de los conceptos del modelo de adaptación de Callista Roy. Aquichán, 2(1), 19-23.

- Dornelles Prolla, C., Santos da Silva, P., Brinckmann Oliveira Neto, C., Goldim, J. \& Asthon-Prolla, P. (2015). Conocimiento del cáncer de mama y cáncer de mama hereditario en el personal de enfermería de un hospital público. Revista Latino-Americana de Enfermagen, 23(1), 90-7.

- García Sánchez, L.V., Parra Gómez, C.E. \& Insuasty Enríquez, J. (2011). Experiencias de vida en mujeres con cáncer de mama en quimioterapia. Revista Colombiana de Psiquiatría, 40(1). Recuperado de http://www.redalyc.org/articulo.oa?id=80619286002.

- Gill, M. J. (2014). The Possibilities of Phenomenology for Organizational Research, Organizational Research Methods, 17(2), 118-137. Recuperado de sagepub.com/journalsPermissions.nav. DOI: 10.1177/1094428113518348orm.sagepub.com.

- Gutiérrez, M. (2007). Adaptación y cuidado en el ser humano: una visión de enfermería. Bogotá, Colombia: Manual Moderno.

- Instituto Nacional de Estadística, Geografía e Informática [INEGI]. (2012). Estadísticas a propósito del día mundial contra el cáncer de mama, Nayarit. Recuperado de http://www.inegi.org.mx/contenidos/espanol/prensa /Contenidos/estadisticas/2012/cancer18.doc. 
- Lewis, L.F. (2014). Caregivers' experiences seeking hospice care for loved ones with dementia. Qualitative Health Research, 24(9), 1221-1231. Recuperado de http://journals.sagepub.com/doi/abs/10.1177/1049732 $\underline{314545888 .}$

- Melo, E., da Silva, R., Mota, R., Mamede, M., Linard, A. \& Fernandez, A. (2011). Orientación sistematizada de enfermería: instrumento para la recuperación de mujeres en el posoperatorio de mastectomía. Evidentia, 8(35). Recuperado de www.indexf.com/evidentia/n35/ev7431.php.

- Melo, R., Lopes, M., Araujo, T. \& Gimenez Galvão, M. (2011). Aplicación del modelo de adaptación de Roy a un cliente pediátrico en el ambiente hospitalario. Cultura de los cuidados, 0(29), 74-81. DOI: https://doi.org/10.7184/cuid.2011.29.08.

- Organización Mundial de la Salud [OMS]. (2016). Cáncer. Ginebra: OMS. Recuperado de www.who.int/cancer/es.

- Raile Alligood, M. \& Marriner Tomey, A. (2011). Modelos y teorías en enfermería, Barcelona. España: Elsevier Mosby.

- Robles-Castillo, J., Ruvalcaba-Limón, E., Maffuz, A. \& Rodríguez-Cuevas, S. (2011). Cáncer de mama en mujeres mexicanas menores de 40 años. Ginecología Obstetricia de México, 79(8), 482-488.

- Rodríguez, S.M., Viana, T.C. \& Andrade, P.G. (2015). A vida da mulher após a mastectomía á luz da teoria adaptativa de Roy. Journal of Research Fundamental Care [on line], 4(7), 3292-3304.

- Sánchez, B. (2000). Fenomenología: un método de indagación para el cuidado de enfermería. En Grupo de Cuidado (Ed). Cuidado y Práctica de Enfermería. 2436. Bogotá, Colombia: Universidad Nacional de Colombia.

- Secretaria de Salud [SSA]. (2015). Información estadística de cáncer de mama y cáncer cérvico uterino. Bogotá: SSA. Recuperado de https://www.gob.mx/salud/acciones-yprogramas/informacion-estadistica.

- Tiddlie, Ch. \& Yu, F. (2007). Mixed Methods Sampling: A typology with examples. Journal of Mixes Methods Research, 1(1), 77-100.

- Vivar, G.C. (2012). Impacto psicosocial del cáncer de mama en la etapa de larga sobrevivencia: propuesta para un plan de cuidados integral para supervivientes. Atención Primaria, 44(5), 288-292. 